\title{
Identificación de megatendencias para la integración de Tecnologías de Información en turismo
}
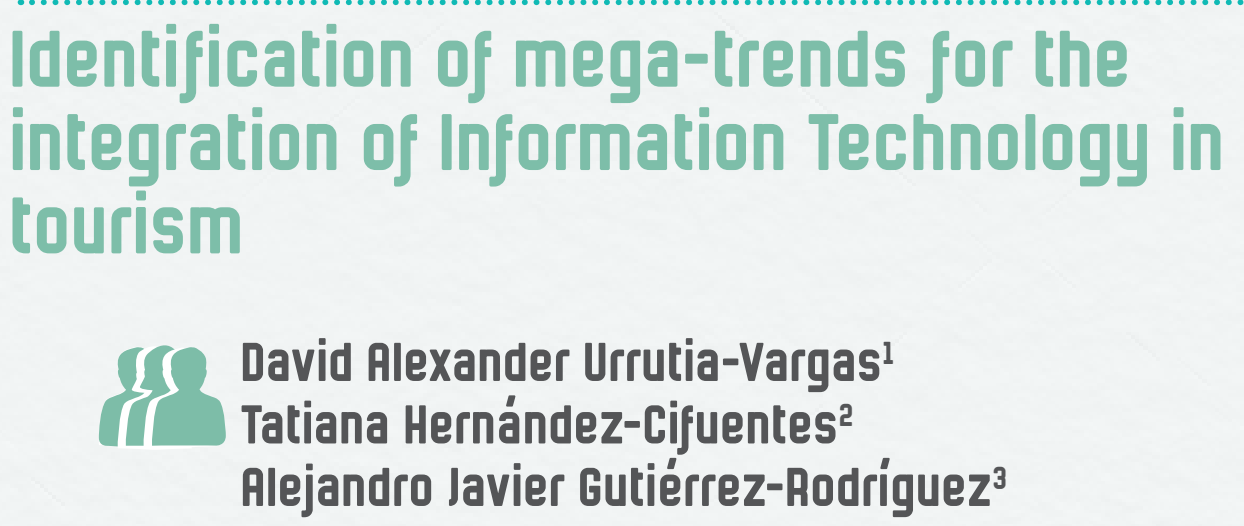

( Recepción: 17/09/2019

( Aprobación: 18/10/2019

( Publicación: 19/12/2019

Para citar este artículo:

Urruria-Vargas, D. A., Hernández-Cifuentes, T., \& Gutiérrez-Rodríguez, A. J. (2019). Identificación de megatendencias para la integración de Tecnologías de Información en turismo. Indagare, (7), 19-27. https://doi.org/10.35707/ indagare/703

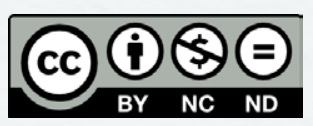

\footnotetext{
${ }^{1}$ Grupo de investigación UNIDERE, Universidad de Ibagué, ORCID: 0000-0002-0063-5925, 1420161007@ estudiantesunibague.edu.co

${ }^{2}$ Grupo de investigación UNIDERE, Universidad de Ibagué, ORCID: 0000-0002-9992-3176, tatiana.hernandez@ unibague.edu.co

${ }^{3}$ Grupo de investigación UNIDERE, Universidad de Ibagué, ORCID: 0000-0001-8866-5514, alejandro.gutierrez@ unibague.edu.co
} 


\title{
Resumen
}

En las organizaciones actuales, las Tecnologías de la Información se reconocen como un aliado no solo para conectarse con grupos de interés externos, sino también para ejecutar operaciones internas. Considerando que la industria turística no es ajena a esta situación, el presente artículo muestra avances en la identificación de tendencias para la integración de tecnologías en la logística demandada dentro de la prestación de servicios turísticos a nivel internacional, lo cual es producto de un ejercicio de vigilancia tecnológica.

\begin{abstract}
In current organizations, Information Technology is recognized as an ally not only to connect with external stakeholders, but also to execute internal operations. Considering that the tourism industry is concerned by this situation, this article shows progress in identifying trends for the integration of technologies in the logistics demanded within the provision of tourism services at international level, which is a product of a technology monitoring exercise.
\end{abstract}

Palabras claves: Tecnologías de la Información, integración de TI, tendencias en TI, logística de servicios, turismo.

Key words: Information Technologies, IT integration, IT trends, service logistics, tourism.

\section{Introducción}

Las Tecnologías de la Información (TI) han ido incrementando su relevancia en las tareas y responsabilidades de los seres humanos y las organizaciones que estos administran. De ahí, que su rol dentro de las empresas esté creciendo y en algunos casos sea de carácter vital para las actividades o funciones de estas (Causado, García, Martínez, \& Herrera, 2015). En materia organizacional, las TI se reconocen como apoyo en la ejecución de operaciones propias del negocio principal de las empresas, así como también se han constituido en un factor clave para administrar efectivamente las relaciones de las empresas con los actores de los diferentes eslabones de la cadena de suministro (Ballou, 2004).

Lo anterior, sin duda alguna, acoge a la industria turística; por lo tanto, es preciso recalcar la posición de los consumidores, clientes o usuarios de los hoteles respecto a la implementación de Tecnologías de Información en los procesos operativos del mismo. En la actualidad, las personas asimilan el término de TI al uso de páginas web, redes 
sociales, aplicaciones de turismo y marketing. Partiendo de ello, se logra evidenciar de manera precisa que aquellas personas que requieren y hacen uso del servicio de hotelería, presentan una posición o comportamiento amigable en cuanto al uso e implementación de TI en las áreas del hotel donde necesitan información, comunicación o entretenimiento (Ukpabi \& Karjaluoto, 2017).

Respecto a la información proporcionada con anterioridad, se entiende que la implementación de TI dentro de las funciones y áreas logísticas de los hoteles es una oportunidad para mejorar la eficiencia y eficacia al momento de realizar la prestación de los servicios a los clientes (Gil \& Meroño, 2006). Para ello, Scholochow, Fuchs, \& Höpken (2010) han encontrado tres etapas en las que se generan estas acciones: la primera consiste en los componentes internos del hospedaje, ya que permiten tener un control sobre el número preciso de huéspedes, empleados o equipo de trabajo y recursos (alimentos, entretenimiento, etc.); en la etapa dos se usan las TI como herramienta para conferencias, foros de preguntas y respuestas o alquiler de salas del hotel; mientras que la última etapa consiste en un sistema comparativo entre insumos y salidas de las actividades ejecutadas financieramente.

Partiendo de lo expresado en los diferentes planteamientos, se entiende que las TI tienen una diversidad de aplicaciones dentro de los hoteles y el turismo; muestra de ello, son las tendencias de consumo que dinamizan el mercado de forma global, por ejemplo, se encuentran clientes mucho más informados, capacitados y exigentes, que solicitan experiencias únicas dentro y fuera de sus habitaciones (Angus \& Westbrook, 2018); asímismo, se reconocen los beneficios que su integración puede otorgar a la satisfacción de los mismos (Stankov, Filimonau, \& Slivar, 2018). Para ilustrar esto, se encuentra la creciente tendencia del uso de realidad virtual, con la finalidad de generar emociones positivas en los consumidores respecto a las ofertas turísticas y de hoteles (Bogicevic, Seo, Kandampully, Liu, \& Rudd, 2019).

Se justifica la necesidad de identificar las tendencias para la integración y aplicación de TI dentro de la industria turística, por cuanto la integración de las mismas se concibe como un factor clave para desarrollar ventajas competitivas (Teymouri \& Ashoori, 2011). Lo anterior se ratifica a través de la revisión de literatura, en la cual se evidencia que se han ejecutado diversas investigaciones que han tenido éxito en la búsqueda del uso y aplicación en herramientas tecnológicas en las áreas funcionales de los hoteles y agentes de turismo hasta la presente fecha. 
En Croacia, Šerić \& Gil (2011) realizaron un estudio en hoteles de alta gama respecto a la satisfacción del usuario con la experiencia propuesta por el hotel, al hacer uso de estas herramientas. Asimismo, en España se ejecutó un estudio de percepción respecto a la relación del administrador o agente de turismo frente la integración y aplicación de TI en procesos y áreas de función (Infante Moro, Infante Moro, Martínez López, \& García Ordaz, 2016).

En ese mismo sentido, se pueden mencionar otras modalidades en que estas herramientas pueden innovar dentro del sector turístico; por ejemplo, en Grecia se realizó un estudio que buscaba, mediante estas tecnologías, generar empleos y recursos para la ciudadanía, para tener una mejor calidad de vida (Sarantopoulos, Vicky, \& Geitona, 2014). Adicional a ello, se han llevado a cabo investigaciones en China que han expresado que desde la década de los ochenta es uno de los países con mayor índice de crecimiento y lugar turístico respecto a la acomodación y hotelería; por ende, hacen énfasis especial en la integración de TI para lograr avances en industria y tecnología para sus hoteles; y el impacto que esto genera en el desarrollo de este mercado (Xiaoqiu Ma, Buhalis, \& Song, 2003).

La realización de una investigación de esta índole permite comprender a profundidad el impacto que ejercen las herramientas de TI en los procesos de desarrollo y función interno de los actores del sector, así como la oportunidad de apreciar las tendencias en apogeo, que influencian el comportamiento y decisiones de consumo de los usuarios (Mihalic \& Buhalis, 2013). Por otro lado, se debe entender que el sector hotelero es complejo, diverso y competitivo, puesto que manejan diversas variables como servicio alimenticio, de entretenimiento, modalidades de pago y la seguridad tanto personal como de bienes; por ende, el conocimiento de estas herramientas se hace significativo; ya que esto influye en la calidad de la prestación del servicio y, por ende, en la concepción del consumidor al observar esto (Nicolae Jucan \& Dolf Baier, 2013). De igual manera, identificar dichas herramientas, permite entender qué ocurre en el macroentorno del mercado donde se desenvuelven los hoteles, y así poder estar a la vanguardia en lo que necesitan los clientes (Bethapudi, 2013).

\section{Materiales y métodos}

El estudio se cataloga en el marco de una investigación mixta al combinar factores de carácter cualitativo y cuantitativo (Hernández, Fernández, \& Baptista, 2010), cuyo enfoque es de carácter descriptivo, debido a la observación de cada componente del estudio como las herramientas, sus causas, los efectos, actores involucrados y la naturaleza de cada uno. De acuerdo con lo expresado, el alcance metodológico es deductivo, dado 


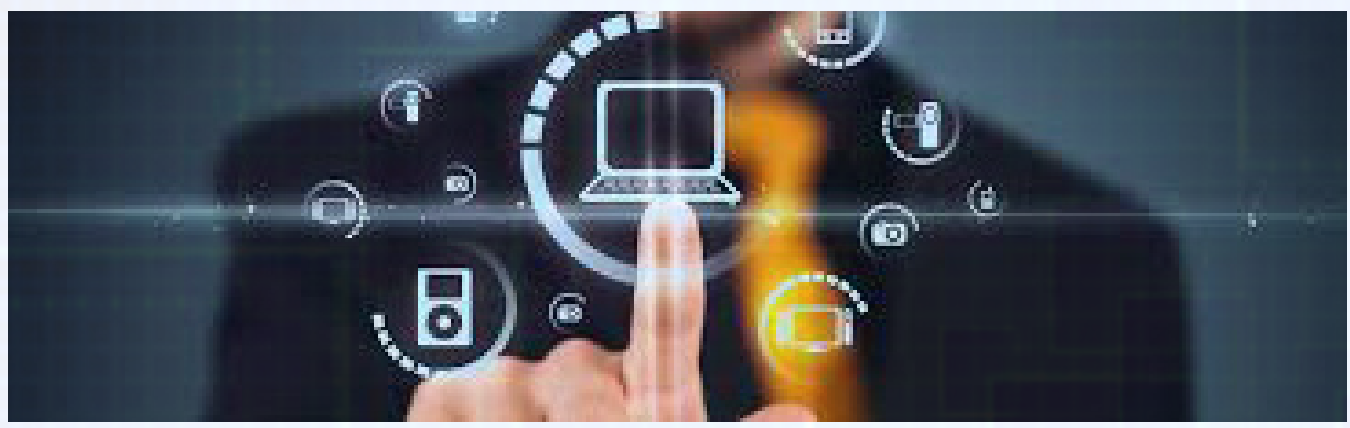

que se parte del problema específico del estudio y culmina con la identificación de las tendencias tecnológicas que están siendo integradas en la industria turística de forma exitosa a nivel internacional (Arango, Tamayo \& Fadul, 2012).

Para dicho proceso se lleva a cabo un ejercicio de vigilancia tecnológica compuesto por los siguientes cuatro pasos: Definición de necesidades, búsqueda y recogida de información, tratamiento de la información y, por último, la difusión y protección de la información (ANAIN, 2008). Por otro lado, se entiende como sujeto de estudio al sector turístico a nivel internacional, en particular los prestadores de servicios hoteleros (Ruiz, Gil, \& Moliner, 2013).

Partiendo de ello, se procede en la obtención de información necesaria y requerida para el estudio; teniendo en cuenta que la forma cómo se desarrollan las ideas y datos recopilados puede llevar a un mejor entendimiento del espectro u objetivo del estudio (Morcillo, 2003). Esto con la finalidad de identificar las tendencias que dinamizan la manera en que se comportan los consumidores según sus nuevos deseos y los procesos internos de los oferentes, que llevan al mercado a comportarse de nuevas y modernas formas (Arango et al., 2012).

De acuerdo al proceso metodológico definido por ANAIN (2008), la identificación de necesidades parte desde la comprensión de la cadena de valor que presenta un hotel, los actores involucrados, las estrategias de organización que implementan y los antecedentes que puedan existir; en líneas generales, sería un entendimiento del funcionamiento, estructura y resultados que un hotel produce al llevar a cabo su cadena de valor.

Para la búsqueda y recolección de información, se consideran los grados de complejidad de las herramientas de búsqueda, la tipología, sus características y los costos que esto puede implicar (ANAIN, 2008). Dicha información se recolecta mediante fuentes secundarias (Fernández, 2008), teniendo como soporte literatura académica que relacione información respecto a la actualidad de las TI y sus tendencias en el turismo, así como los registros de patentes vinculadas al tema (Arango et al., 2012). 
Dentro del tratamiento de la información, ANAIN (2008) destaca tareas tales como la credibilidad de la fuente, la modalidad de obtención de esos datos, la concordancia entre fuente e información, y la validación de esa información a través de agentes externos.

Finalmente, este ejercicio de vigilancia tecnológica supone la difusión y protección de dicha información; de tal forma que se puedan compartir a los hoteles de la región el análisis de las megatendencias identificadas (ANAIN, 2008). Después de tratar los datos obtenidos y haber hecho su respectivo procesamiento, se procederá a difundir las conclusiones alcanzadas durante la investigación, dando así las sugerencias respectivas a los agentes involucrados y la respuesta tanto al problema como a la pregunta de investigación planteados en un principio.

\section{Resultados parciales}

En primer lugar, se definieron las palabras que delimitarían la ruta de acceso; en ese sentido, se definió como palabra clave de primer nivel Service logistics por cuanto el proyecto pretende hacer hincapié sobre las operaciones requeridas para prestar un servicio, en este caso, de alojamiento. El seguimiento a las patentes se realizó a través de las bases de datos de Scopus, Web of Science, Google Patents, Patent Inspiration, así como las oficinas de patentes de Estados Unidos y de la Unión Europea.

Una vez efectuada la búsqueda por medio de la palabra clave de primer nivel, se identificó la necesidad de filtrar la búsqueda de manera tal que los resultados que arrojara correspondieran con la industria y el sector objeto de estudio en esta propuesta, por lo tanto, se filtraron los documentos con las siguientes palabras y orden:

- Tourism,

- Hotels,

- Information Technology,

- Accommodation.

A continuación, se presenta un consolidado de las patentes encontradas hasta la fecha:

- Dataflow control instruction generation device, dataflow control instruction generation method, and dataflow control instruction generation program.

- Method and system for efficient communication.

- Big data analysis platform for travel and tourism.

- Recommendation device, information terminal, recommendation method, and non-transitory storage medium.

- Resource processing method and device. 
- System and methods for conserving guest room resources and utilities using Internet of Things devices.

- System and methods for enhancing electronic communications.

- Systems and methods for customizing hotel, timeshare, and rental property guest experiences, and conserving resources and utilities using Internet of Things devices and location tracking.

- Travel management system.

- Travel subscription devices and methods.

- Virtual reality content to a virtual reality server by a virtual reality device.

Las patentes mencionadas reflejan que existe una tendencia dentro de la industria de turismo por sistemas de comunicación eficientes, modelos de realidad virtual y control de tareas a distancia, sistemas de recolección de datos según gustos mostrados en redes sociales y, sobre todo, el uso de comunicación electrónica en procesos internos y externos con los usuarios.

\section{Potencial uso}

La comprensión de las megatendencias es un factor clave de diferenciación que buscan tener las empresas actuales dentro del mercado que operan, debido a la variedad de competidores que pueden cautivar, atraer y atrapar el catálogo de clientes que se tenga a disposición. En ese mismo sentido, las TI cuentan con una participación considerable en el desarrollo de la industria donde se empleen, de forma tal que contribuyen en cada una de las áreas funcionales de las empresas.

Por ende, los productos del ejercicio de vigilancia tecnológica permiten ser contrastados con otros objetivos de la investigación, en los cuales se busca diagnosticar el nivel de integración actual de Ti en el sector hotelero del Tolima. De manera tal que se puedan identificar estrategias que contribuyan al desarrollo tecnológico de un sector emergente en la región como lo es el turismo, lo anterior con miras a reducir el rezago tecnológico que existe frente al nivel internacional.

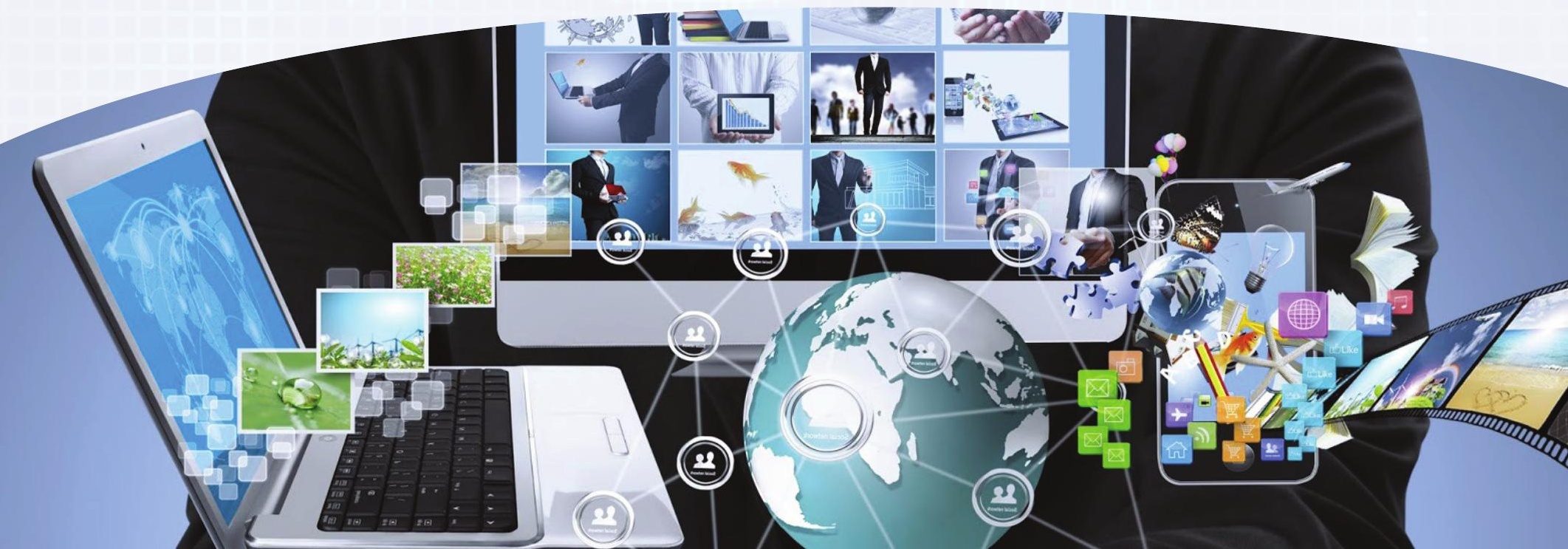




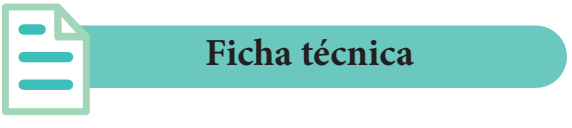

Título del proyecto:

Actualización estratégica del Clúster de Turismo (Ibagué - Tolima).

PRIT: Agroindustria innovadora de base tecnológica.

Código del proyecto: 19-509-INT.

Palabras claves: Clúster, estrategia, turismo.

Grupo de investigación: UNIDERE.

Investigador principal: Alejandro Javier Gutiérrez Rodríguez.

Correo electrónico: alejandro.gutierrez@unibague.edu.co

\section{Referencias}

ANAIN. (2008). Guía práctica. La gestión de la Innovación en 8 pasos. ANAIN - Agencia Navarra de Innovación, 104.

Angus, A., \& Westbrook, G. (2018). Las 10 principales tendencias globales de consumo para 2019. Euromonitor Internacional, 43.

Arango Alzate, B., Tamayo Giraldo, L., \& Fadul Barbosa, A. (2012). Vigilancia tecnológica: Metodologías y aplicaciones. Revista Gestión de las Personas y Tecnología, 5 (13), 250-261. Recuperado de http://www.revistas.usach.cl/ojs/index.php/revistagpt/article/view/615

Ballou, R. (2004). Logística: Administración de la Cadena de Suministro. (E. Quintanar, Ed.) (Fifth). Naucalpan de Juárez: Pearson Educación.

Bethapudi, A. (2013). The Role of ICT in Tourism Industry. Journal of Applied Economics and Business, 1(4), 67-79. Recuperado de http://www.aebjournal.org/articles/0104/010406.pdf

Bogicevic, V., Seo, S., Kandampully, J. A., Liu, S. Q., \& Rudd, N. A. (2019). Virtual reality presence as a preamble of tourism experience: The role of mental imagery. Tourism Management, 74, 55-64. https://doi.org/10.1016/j.tourman.2019.02.009

Causado Rodríguez, E., García Guiliany, J., Martínez Ventura, J., \& Herrera Flórez, A. (2015). Tecnologías de información y comunicación. Barranquilla: Centro de investigación y proyectos (CINPRO), Coorporación Universitaria Latinoamericana.

Gil Corbalán, N., \& Meroño Cerdán, A. (enero, 2006). Efectos del empleo de las tecnologías de la información en hoteles. En VI Congreso Turismo y Tecnologías de la Información y las Comunicaciones, Turitec.

Hernández, R., Fernández, C., \& Baptista, M. (2010). Metodología de la Investigación. (J. Mares, Ed.) (5th ed.). México D.F.: McGRAW-HILL. Recuperado de https://institutoprofesionalmr. org/wp-content/uploads/2018/04/Hernández-Fernández-Baptista-2010-Metodologia-de-laInvestigacion-5ta-edicion.pdf

Infante Moro, A., Infante Moro, J. C., Martínez López, F. J., \& García Ordaz, M. (2016). Percepción de la importancia de la seguridad informática en la industria hotelera española. En IX Jornadas Investigación en turismo. El turismo y la experiencia de cliente (Tomo II). Recuperado de 
https://www.researchgate.net/publication/305442975_Percepcion_de_la_importancia_de_la_ seguridad_informatica_en_la_industria_hotelera_espanola

Mihalic, T., \& Buhalis, D. (2013). ICT as a new competitive advantage factor-case of small transitional Hotel Sector. Economic and Business Review, 15(1), 33-56. Recuperado de http://ojs.ebrjournal. net/ojs/index.php/ebr/article/view/214

Morcillo, P. (2003). Vigilancia e inteligencia competitiva: fundamentos e implicaciones. Revista Madri+d, (17), 15-21.

Nicolae Jucan, C., \& Dolf Baier, M. S. (2013). The ICT Implication on CSR in the Tourism of Emerging Markets. Procedia Economics and Finance, 3(12), 702-709. https://doi.org/10.1016/s22125671(12)00217-1

Ruiz Molina, M. E., Gil Saura, I., \& Moliner Velázquez, B. (2013). Technologies that hotels implement - technologies that guests like. Revista de Análisis Turístico, 61-70. Recuperado de https://aecit. org/jornal/index.php/AECIT/article/view/144

Sarantopoulos, L., Vicky, K., \& Geitona, M. (2014). A Supply Side Investigation of Medical Tourism and ICT Use in Greece. Procedia - Social and Behavioral Sciences, 148, 370-377. https://doi. org/10.1016/j.sbspro.2014.07.055

Scholochow, C., Fuchs, M., \& Höpken, W. (2010). ICT Efficiency and Effectiveness in the Hotel Sector. Information and Communication Technologies in Tourism, 13-24. https://doi.org/10.1007/978-3211-99407-8_2

Šerić, M., \& Gil Saura, I. (2011). Análisis de los hoteles de alta categoría de croacia desde el enfoque hotel-huésped. Investigaciones Europeas de Dirección y Economía de la Empresa, 17(3), 127-149. https://doi.org/10.1016/S1135-2523(12)60124-5

Stankov, U., Filimonau, V., \& Slivar, I. (2018). International Journal of Hospitality Management Calm ICT design in hotels : A critical review of applications and implications. Hospitality Management, 82, 298-307. https://doi.org/10.1016/j.ijhm.2018.10.012

Teymouri, M., \& Ashoori, M. (2011). The impact of information technology on risk management. Procedia Computer Science, 3, 1602-1608. https://doi.org/10.1016/j.procs.2011.01.056

Ukpabi, D. C., \& Karjaluoto, H. (2017). Consumers' acceptance of information and communications technology in tourism: A review. Telematics and Informatics, 34(5), 618-644. https://doi. org/10.1016/j.tele.2016.12.002

Xiaoqiu Ma, J., Buhalis, D., \& Song, H. (2003). ICTs and Internet adoption in China's tourism industry. International Journal of Information Management, 23(6), 451-467. https://doi.org/10.1016/j. ijinfomgt.2003.09.002 